\title{
Anabases
}

ANABASES Traditions et réceptions de l'Antiquité

$23 \mid 2016$

Varia

\section{Proche-Orient : patrimoines en grand danger}

\section{Annie Sartre-Fauriat}

\section{OpenEdition}

Journals

Édition électronique

URL : http://journals.openedition.org/anabases/5618

DOI : 10.4000/anabases.5618

ISSN : 2256-9421

\section{Éditeur}

E.R.A.S.M.E.

\section{Édition imprimée}

Date de publication : 2 mai 2016

Pagination : 139-156

ISSN : 1774-4296

\section{Référence électronique}

Annie Sartre-Fauriat, «Proche-Orient : patrimoines en grand danger», Anabases [En ligne], 23 | 2016,

mis en ligne le 02 juin 2019, consulté le 21 octobre 2019. URL : http://journals.openedition.org/ anabases/5618; DOI : 10.4000/anabases.5618 


\section{Proche-Orient: patrimoines en grand danger}

Annie Sartre-Fauriat

es guerres n'ont jamais été des périodes favorables à la conservation du patrimoine. Faire la liste des villes détruites, des monuments rayés de la carte, des - trésors perdus à jamais obligerait à remonter loin dans le temps. Car la folie guerrière des hommes ne s'est jamais contentée d'exterminer les adversaires, elle s'en prend aussi à leur histoire à travers la destruction d'un patrimoine conservé tant bien que mal au fil du temps. Nous assistons au Proche-Orient depuis des dizaines d'années, au Liban, en Iraq, au Yémen et en Syrie, non seulement à des catastrophes humanitaires, mais aussi à des catastrophes culturelles par la destruction de villes historiques, de sites archéologiques et de musées. Avec ces destructions c'est tout un pan de l'histoire de l'humanité qui disparaît dans ces pays qui en étaient des conservatoires par leur histoire millénaire et l'importance des vestiges qui y étaient visibles. Bombardements, pillages et vandalisme sont en train non seulement de faire disparaître des monuments témoins du passé, mais aussi de priver l'humanité des maillons de son histoire en arrachant au sol des objets dont seul le contexte permet la compréhension.

Toutefois, jamais encore, on n'avait assisté à une telle folie destructrice volontaire du patrimoine comme celle à laquelle s'est livré le groupe Daech en Iraq et en Syrie depuis 20I4.

Bien que la convention de La Haye de i954, complétée par le protocole de i999, signée par la plupart des pays du monde, stipule que « les biens culturels immeubles ne doivent pas être utilisés à des fins militaires », les combats qui se sont déroulés ces dernières vingt années au Proche-Orient ont constamment violé la convention en se déroulant au cœur même des sites historiques. Ainsi, en Iraq en rg9ı puis en 2003, l'armée américaine a allègrement participé aux destructions en installant par 
exemple son camp “Alpha» dans les ruines de Babylone (fig. I). Quand, en 2oog, le Comité international de coordination de l'unEsco pour la sauvegarde du patrimoine culturel de l'Iraq a dressé la liste des déprédations causées par cette installation, on a pu constater que le site avait été nivelé au bulldozer pour construire une aire de stationnement de véhicules lourds et une piste d'atterrissage pour les hélicoptères. Des constructions de remblais de protection avaient utilisé des poteries et des briques gravées de caractères cunéiformes pulvérisées au préalable. Des tranchées avaient été creusées dans les ruines pour y stocker des réserves de carburant et, sur la fragile voie sacrée du vi ${ }^{\mathrm{e}}$ siècle av. J.-C., des chars avaient brisé les dalles et causé des dommages irréparables aux couches archéologiques et à des milliers d'objets, dont des tablettes gravées, sans oublier les dégâts faits à l'enceinte et à la porte d'Ishtar. Le même constat s'est imposé pour plusieurs sites de Basse-Mésopotamie, comme Tell al-Obeid, occupé par les troupes irakiennes, ou Eridu et Ur par les troupes de la coalition. Dans ce dernier site, les tirs de roquettes avaient gravement endommagé la ziggourat de la déesse Nana, datant de la fin du III $^{\mathrm{e}}$ millénaire, tandis que les jeeps escaladaient les vestiges.

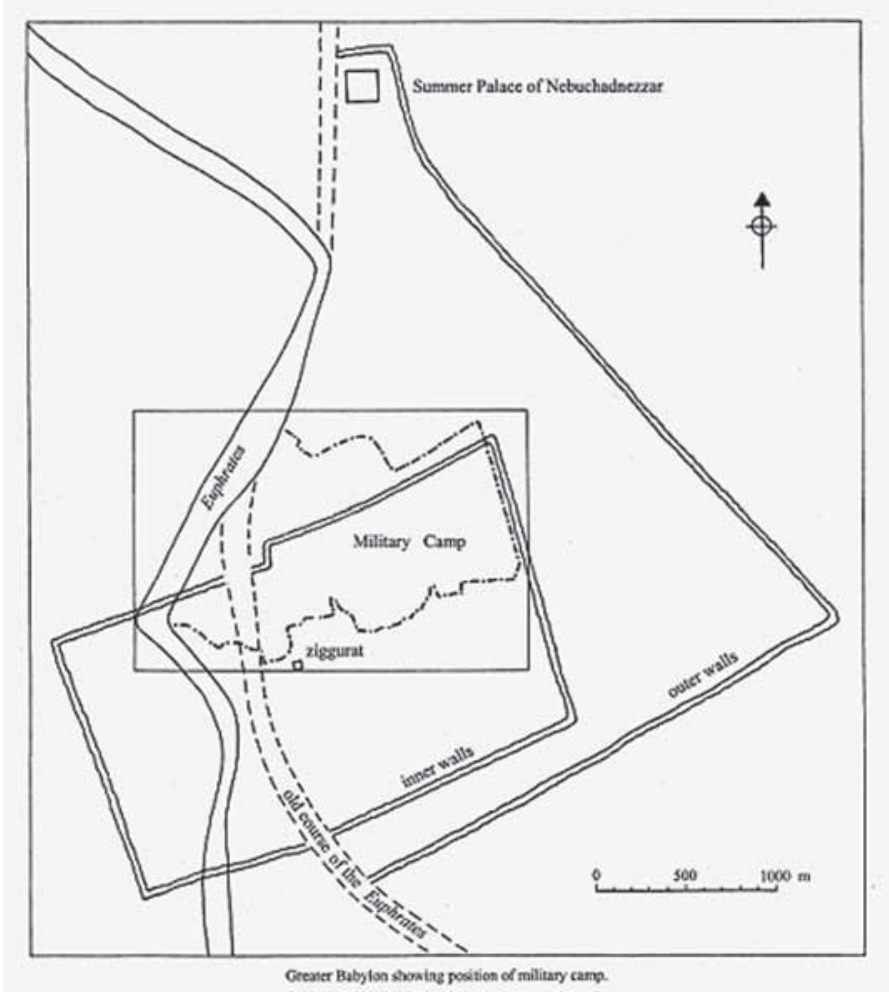

Fig. I- En pointillé, le camp alpha des Américains sur le site antique de Babylone (Iraq). 
Aux mêmes causes les mêmes effets en Syrie depuis le début du conflit, en 20II, qui oppose le régime dictatorial des Assad et les groupes rebelles à cette oppression. Depuis cette date, les combats entre les différents belligérants ont porté atteinte plus ou moins gravement à plusieurs sites historiques. Plus de trois cents sites, sur plusieurs milliers que compte le pays, sont déjà plus ou moins sérieusement endommagés. Jusqu'à l'été 20I5, celui qui avait le plus souffert était Alep, dont la vieille ville est classée au patrimoine mondial depuis ig86. À ce jour, plus de I35 bâtiments y ont été endommagés dont 22 totalement détruits, réduits à des gravats par les tirs de mortier, les bombes et les barils de TNT largués par l'armée du régime Assad ou par les explosions dans des tunnels creusés sous les bâtiments par les opposants afin d'en déloger les soldats du régime. Ainsi, une grande partie des souqs du XIv siècle, parmi les plus beaux du Moyen-Orient, a brûlé en 2012 (fig.2). Sur les vingt-neuf souqs, huit sont totalement détruits, ce sont les souqs Aqqadin, Bezeriyah, Dra (vêtements), el-Haraj (tapis), Manadil, New Istanbul, Siyyagh (des bijoux), Qawouqiliya; seize sont très sévèrement endommagés dont celui d'Atarin (aux épices), Aslan Dada (maroquinerie), Attiqa (cuir), Batiya, Ebi, etc.; ı5oo à ı 6oo échoppes ont disparu, les voûtes des ruelles se sont effondrées et plusieurs khans (entrepôts) ou hammams (bains) qui s'y trouvaient ont été détruits, le khan al-Sabun (du savon) du xvi ${ }^{\mathrm{e}}$ siècle, Fattayin (des fourrures) et Nasser, ou sévèrement abîmés comme an-Nahasin du xiII siècle (chaussures), Burghul de I/722 (blé), al-Oulabiyeh avec ses boiseries peintes d'époque ottomane. La grande mosquée des Omeyyades n’a pas été épargnée. Son minaret du $\mathrm{xI}^{\mathrm{e}}$ siècle a été abattu en 20I3, le mur oriental et le pavement de marbre de la cour ont été gravement endommagés, l'intérieur pillé et le musée des manuscrits incendié. La citadelle du XIII $^{\mathrm{e}}$ siècle, occupée alternativement par l'armée du régime et les opposants, a subi elle aussi des dommages, dont parmi les plus importants l'effondrement d'une section des remparts en juillet 20I5. Mais, plus grave, la plupart des bâtiments qui s'étendaient à ses pieds ont été totalement rayés de la carte à la suite de bombardements aériens et d'explosions souterraines (fig.3). Parmi eux, la mosquée Khusruwiyeh du xvi ${ }^{\mathrm{e}}$ siècle, attribuée à l'architecte turc Sinan, la madrasa Sultaniyeh du xiII ${ }^{\mathrm{e}}$ siècle qui abritait le tombeau du fils de Saladin, gravement détériorée en août 20I4 et qui a totalement explosé en décembre de la même année, ou encore le hammam Yalbugha an-Nasri de la fin du $x^{\mathrm{e}}$ siècle dont les coupoles s'étaient effondrées en août 20I4 et qui a été intégralement détruit le mois suivant. Parmi les quarante-huit autres bâtiments sévèrement endommagés à Alep, on compte la plupart des mosquées de la vieille ville, le grand sérail d'époque ottomane, le bimaristan (hôpital) Argoun du xrv siècle, victime en outre de vandalisme, le khan du tabac du XIII ${ }^{\mathrm{e}}$, le khan al-Wazir, etc. Les bombardements dont Alep est la cible permanente par les forces du régime et les combats au sol contre les rebelles ont eu raison de nouveaux bâtiments depuis janvier 2015 dont, entre autres, le sanctuaire du Cheikh Mohamed Nabhan dans la mosquée Kiltawiye à 
Bab al-Hadid, l'église arménienne des Quarante Martyrs dans le quartier Jdeydeh, qui a par ailleurs subi de nombreuses autres destructions.

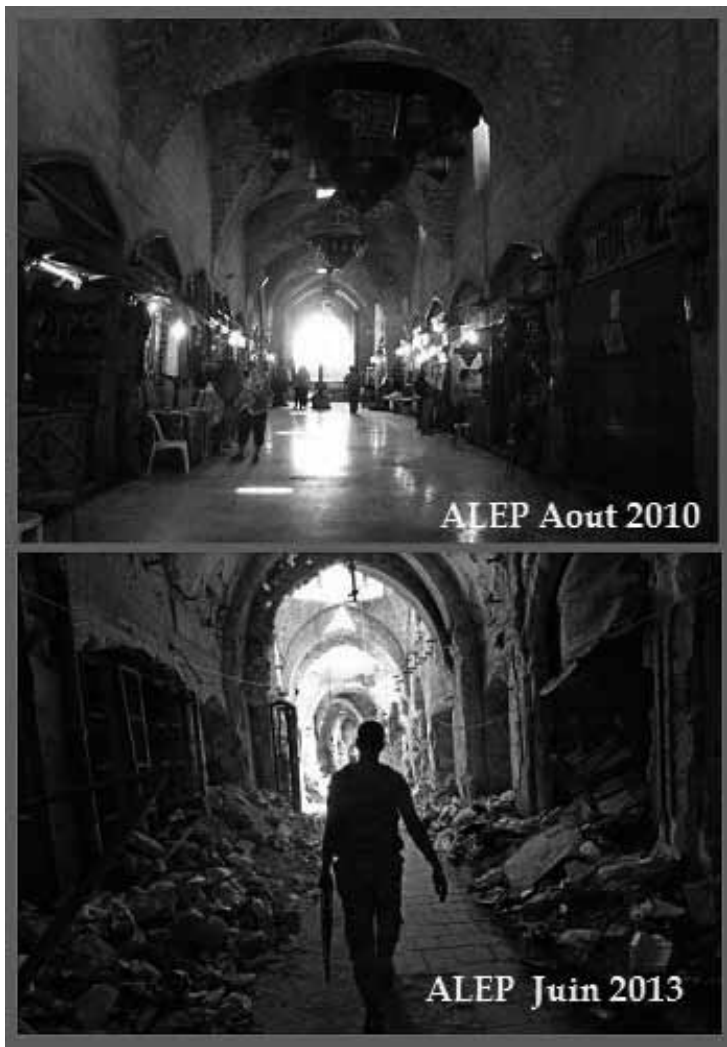

Fig. 2 - Alep. Les souqs avant l'incendie en 2010 et après en 2013.

Trois autres sites classés au patrimoine mondial ont connu eux aussi des destructions ${ }^{1}$. À Bosra dans le Hauran, au sud du pays, la citadelle des $\mathrm{xI}^{\mathrm{e}}-\mathrm{xIII}^{\mathrm{e}}$ siècles qui enserre le théâtre romain, a servi de base de lancement d'obus et de roquettes qui ont touché plusieurs monuments de la ville. Le nymphée a perdu l'architrave qui

1 À ce jour, six sites en Syrie ont été classés au patrimoine mondial par l'Unesco : l'ancienne ville de Damas en i979, Bosra et Palmyre en I980, l'ancienne ville d'Alep en ig86, le Krak des chevaliers et le château de Salah ad-Din (ex de Saône) en 2006 et les villes mortes du nord de la Syrie en 20II. Étaient inscrits sur la liste indicative en vue d'un classement, les norias de Hama, Ougarit, Ebla (tell Mardikh), Mari (tell Hariri), Doura Europos, Apamée, Qasr al-Hayr ach-Charqi, Maaloula, la cité-citadelle croisée de Tartous, la cité abbasside de Raqqa et l'île de Rouad. 
surmontait la colonne d'angle et la reliait au mur de fond, des maisons anciennes alentours ont été détruites et un mur de la cathédrale Saints-Serge-et-Bacchus s'est effondré. Plusieurs minarets de mosquées ont également été endommagés et une route stratégique traverse les structures sud de l'amphithéâtre, non encore fouillé, à l'ouest de la citadelle. Le Krak des chevaliers a été lui aussi gravement affecté par des bombardements. Avant les destructions radicales et volontaires qui ont ému le monde entier, le site de Palmyre avait lui aussi souffert de la présence des armées et des tirs. L’armée du régime, depuis le château de Fakhr ed-Din qui domine le site, tirait sur les opposants embusqués dans l'oasis en contre bas. Le temple de Bêl avait reçu des éclats d'obus, quelques colonnes de la grande colonnade avaient été touchées, tandis qu'une route stratégique, tracée dans la nécropole nord, avait provoqué l'effondrement de plafonds d'hypogées et ébranlé les tours funéraires voisines. Des tranchées pour dissimuler des armements avaient retourné le sol et causé des dommages dont l'ampleur est difficile à apprécier pour le moment (fig.4).

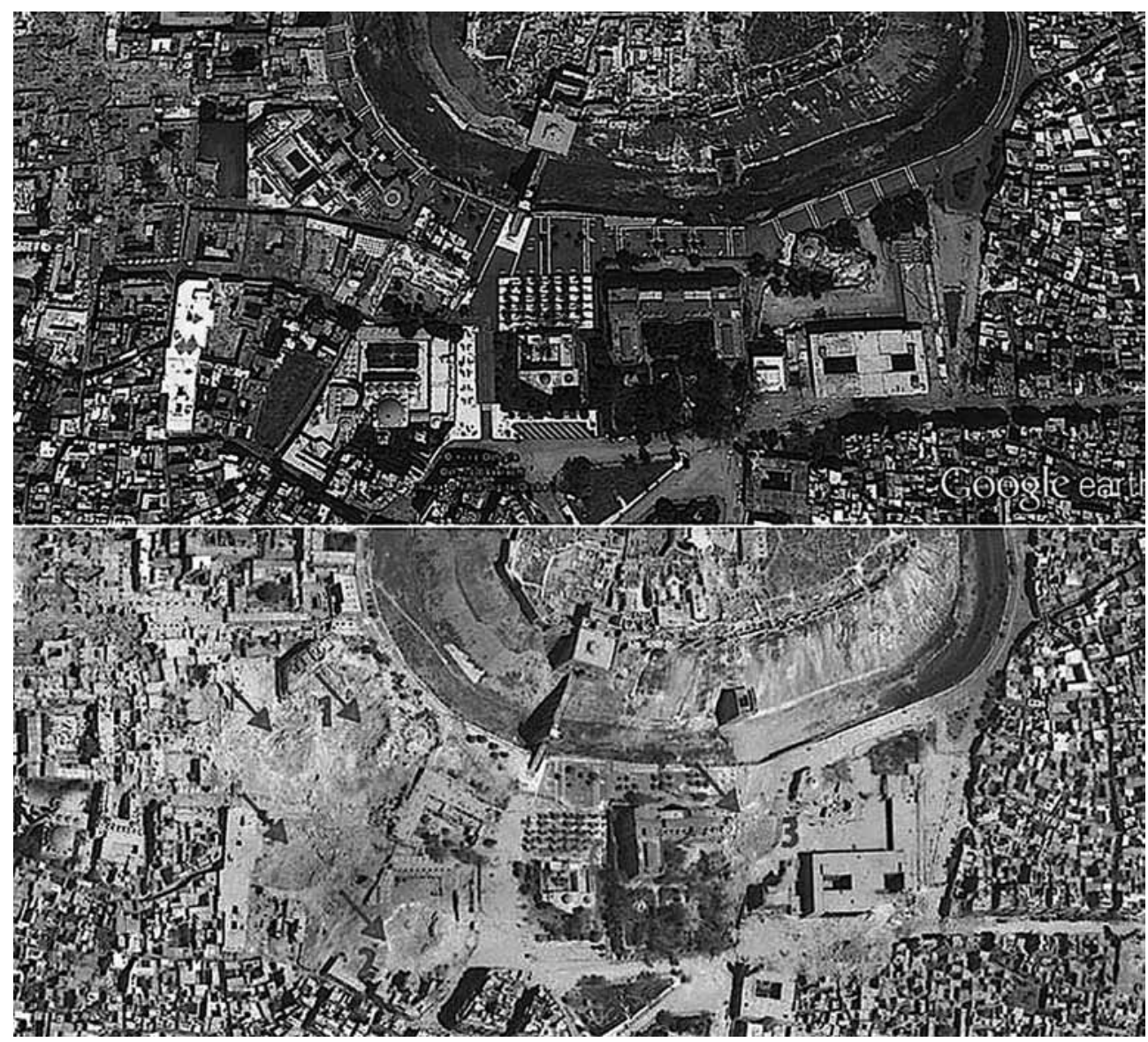

Fig. 3 - Alep. Destructions au pied de la citadelle. 

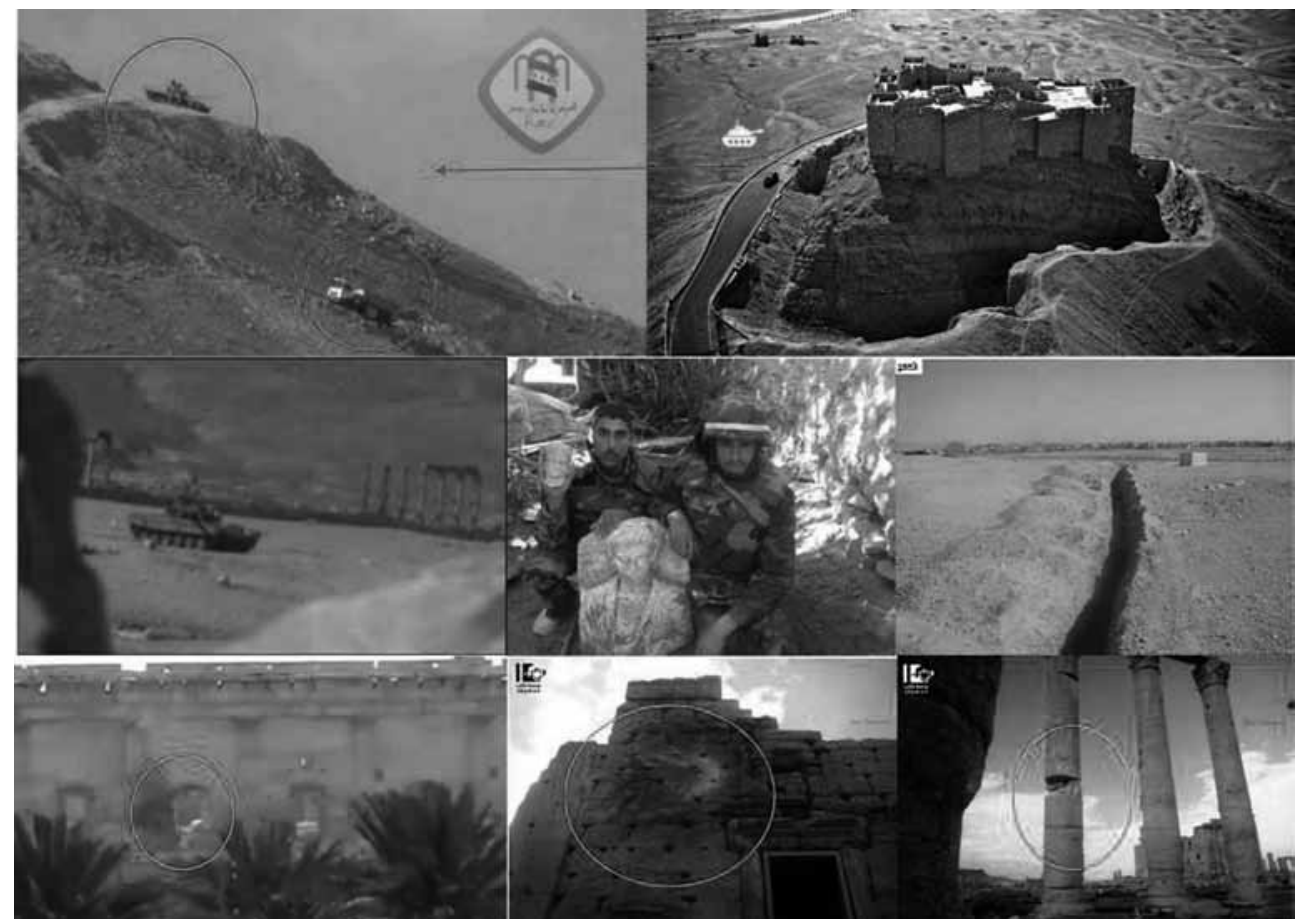

Fig. 4 - Palmyre. Destructions par l'armée du régime entre $201 \mathrm{I}$ et 2015.

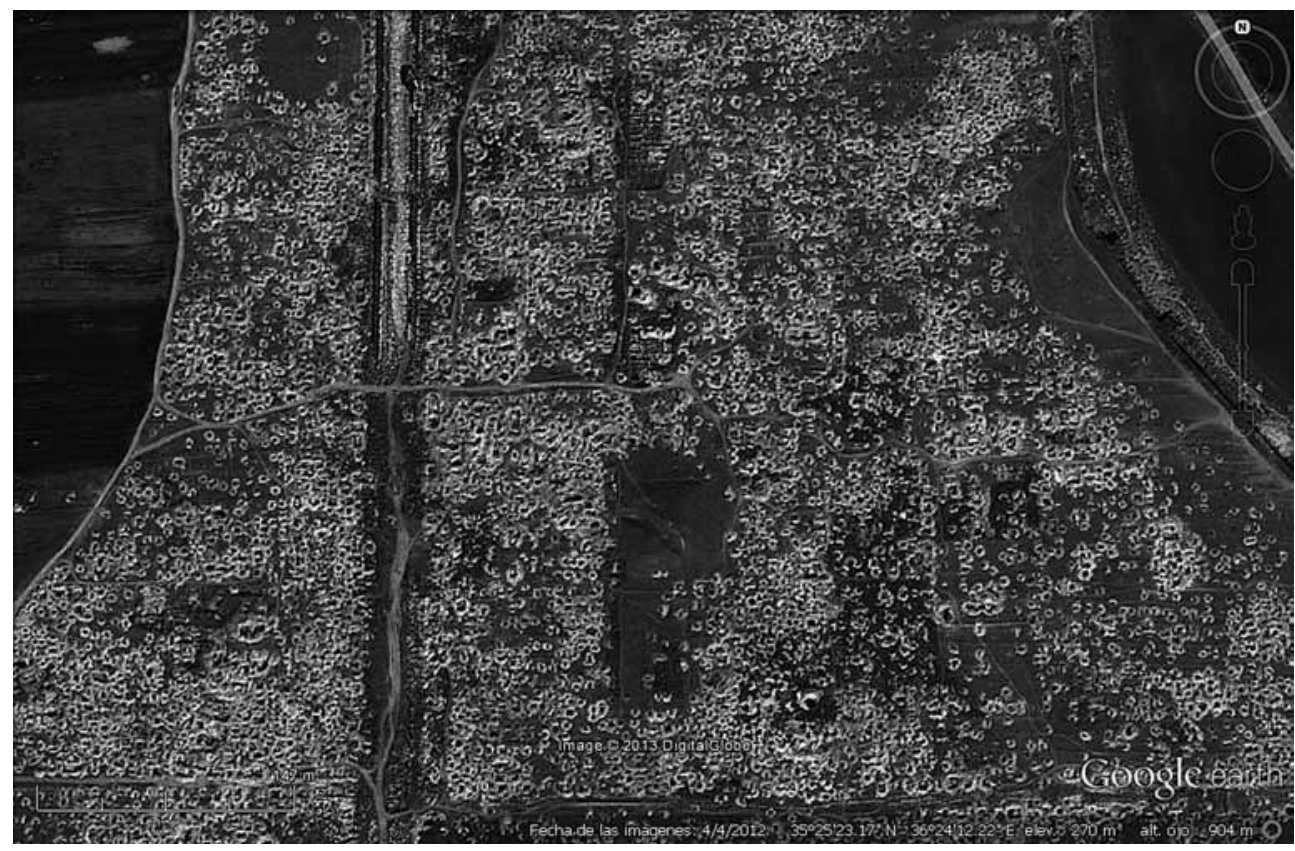

Fig. 5 - Apamée. Trous de pillages de part et d'autre de la grande colonnade. 
On ne peut que constater que, pas plus que le protocole de ig54, celui de i999 qui préconisait d'éviter «de placer des objectifs militaires à proximité des biens culturels » n'est respecté par les parties en présence. L'unesco, qui ne peut intervenir qu'auprès des gouvernements, ne parvient pas à se faire entendre en Syrie et l'arrivée des troupes russes sur le terrain à la fin de l'été 20I5, avec des chars et des avions, ne laisse pas envisager l'arrêt des destructions. Plusieurs villages de Syrie du Nord ont été pilonnés et, à ce jour, n’ont été révélées que les destructions sur le site de Khirbet Hass (Sinshara), mais il y a fort à parier que d'autres vestiges de ces villages de l'époque byzantine, dont certains étaient presque intacts, sont ou seront affectés ${ }^{2}$. Tant que les groupes armés continueront de se battre dans les sites historiques et qu'aucune protection ne sera mise en œuvre, les destructions ne feront que se multiplier.

Comme l'Iraq, dont de nombreux sites ont été dévastés durant les guerres de I99I et de 2003, la Syrie voit progressivement depuis 20II disparaître un patrimoine unique, témoin des civilisations successives dont les deux pays portent la marque. La Syrie, plus encore que l'Iraq, concentre en effet sur son sol toute l'histoire de l'humanité depuis la Préhistoire. Lieu de passage et carrefour des empires et des peuples du Proche-Orient, elle a conservé la trace de chacun. Or ce patrimoine, dont on vient de voir qu'il est mis en grand péril par les combats, est malheureusement affecté aussi par les pillages systématiques pratiqués de «façon industrielle», mais par-dessus tout par les destructions volontaires mises en scène de façon spectaculaire par leurs auteurs.

Le pillage des sites et le trafic illicite d'antiquités contribuent en effet eux aussi très largement à la destruction du patrimoine. Sur ce plan, la Syrie connaît le même sort que l'Iraq dont le sol a été, et continue d'être, labouré et saccagé par les pilleurs $^{3}$. On estime que depuis le départ des Américains, $25 \%$ des sites de BasseMésopotamie ont été détruits par le pillage. Larsa, Umma, Nippur, Isin, mais aussi de nombreux petits sites non encore fouillés, subissent de véritables razzias de la part de bandes organisées, dont certaines arrivent en hélicoptère, et pratiquent un pillage sélectif ciblé sur les sceaux-cylindres et les tablettes. Avec plusieurs milliers de sites archéologiques, l'Iraq et la Syrie ont toujours été des réservoirs d'antiquités exhumées de manière plus ou moins légale. La vallée de l'Euphrate, le Hauran, les sites d'Apamée ou de Palmyre en Syrie sont bien connus pour avoir alimenté le marché de l'art et les musées du monde entier! Mais avec le début

2 Dans la mesure où les Russes aident le régime en place, la DGAM à Damas fait silence, volontaire ou forcé, sur les destructions provoquées par leurs bombardements.

3 Des phénomènes du même ordre sont constatés en Libye où le pillage alimente lui aussi un trafic d'œuvres d'art, notamment sur les sites de Cyrène et d'Apollonia. 
du conflit en 2oII en Syrie, les choses ont pris des proportions gigantesques. Des photos satellites témoignent de ces fouilles illicites entreprises soit par des individus isolés soit par des bandes organisées liées aux mafias internationales. Ces pillages ont particulièrement affecté les sites d'Apamée dans la vallée de l'Oronte, de Palmyre, d'Ebla en Syrie centrale, Doura ou Mari sur l'Euphrate. À Apamée, les photos aériennes sont saisissantes: de part et d'autre de la grande colonnade, on compte plus de I40oo trous de fouilles; des mosaïques protégées par des mètres de béton, ont été volées et déjà vendues (fig.5). Près de 2ooha du site sont soumis aux pillages, et nombre de monuments ont subi des dommages pour des raisons mal identifiées (attaques armées, destructions volontaires?) comme le cardo, l'agora, les bains de Julius Agrippa, les murs d'enceinte, le Tychéion, le marché. À Palmyre, des tombeaux ont fait l'objet de pillages systématiques. C'est le cas, entre autres, des hypogées $\mathrm{F}$ et $\mathrm{H}$ dans la nécropole sud-est, restaurés au début des années 2000 par une équipe d'archéologues de l'université de Nara au Japon, dévalisés dès les débuts du conflit en 20II, et aujourd'hui totalement dévastés. De nombreuses saisies d'objets volés dans des tombes ont été opérées par le service des antiquités (DGAM), plus particulièrement des bustes funéraires ou des bas-reliefs provenant des sarcophages, mais beaucoup manquent à l'appel.

Éventrés au bulldozer et pillés plusieurs tells, comme celui d'Ebla (Tell Mardikh) datant des $\mathrm{II}^{\mathrm{e}}$ et $\mathrm{II}^{\mathrm{e}}$ millénaires, où la zone $\mathrm{F}$ a été déblayée par de gros engins et où tous les temples (Ishtar, Reshaph, Shamash) et les palais (palais nord et palais Q) ont subi de sévères dégradations. Beaucoup d'autres tells partout sur le territoire de la Syrie servent de postes d'observation aux armées qui, non seulement les escaladent avec des auto-chenilles et des tanks, creusent des abris, et construisent des casemates, mais pratiquent des fouilles clandestines. Ainsi dans le Hauran à Tell Ashtarah, Tell al-Ashari (Dion de la Décapole), Tell Shehab; sur l'Euphrate à Tell al-Sinn, Tell Bi'a ou dans la vallée du Khabour à Tell Sheikh Hamad (Dur Katlimmu) et Tell Ajaja. Les sites les plus célèbres n'ont bien sûr pas été épargnés. À Mari, I20o fosses de pillages ont été repérées par satellite sur l'ensemble du site; à Doura-Europos des gangs armés ont pris le relais des populations locales avec des moyens de prospection électronique et ont déjà vendu des fresques, des bijoux, des statues, de la céramique. Aucun monument du site de Doura n’a échappé aux déprédations: palais du dux ripae, temples d'Adonis et d'Artémis, synagogue, nécropole où l'on compte des milliers de trous de pillage. La liste serait longue de tous les sites endommagés ou ravagés. L’occupation des ruines du massif calcaire par des réfugiés ayant perdu leurs maisons, comme à Serdjilla ou à Khirbet Hass où maisons et tombes antiques sont utilisées comme habitat ou comme école, ajoute encore un peu plus aux menaces.

Mais les atteintes les plus révoltantes sont celles qui procèdent de destructions volontaires. De ce point de vue, beaucoup d'acteurs du conflit en portent 
la responsabilité et l'on soupçonne l'armée du régime Assad de s'y être livrée à plusieurs reprises contre des monuments dont l'enjeu stratégique n'était pas majeur, mais qui avaient de l'importance pour les habitants des lieux. C'est ainsi que l'on est fondé à se poser la question pour le musée des mosaïques de Maaret en-Noman, dont les habitants avaient organisé la protection et qui a reçu une bombe larguée par un hélicoptère du régime au printemps 20ı5. Ou bien encore pour les colonnes du nymphée de Bosra et l'ensemble des mosquées sunnites d'Alep dont l'intérêt militaire n'est pas a priori l'essentiel. La raison de leur destruction est davantage à chercher dans la volonté de procéder à des frappes plus punitives que réellement nécessaires stratégiquement, précisément à l'encontre des quartiers ou des villages en rébellion contre le pouvoir dictatorial. Et nous n'insisterons pas sur les pillages auxquels l'armée syrienne s'est livrée en particulier à Palmyre avant d'abandonner le site à Daech. Mais on est actuellement plus particulièrement frappé par les destructions violentes et massives perpétrées au nom d'une idéologie religieuse intolérante. De ce point de vue, parmi les groupes qui se sont livrés à ces actes, celui de Daech est le plus radical. Mais des exactions de même type avaient pu être repérées avant l'arrivée de Daech en Syrie du Nord, et en particulier à El-Bara dans le Jebel Zawwiyeh où des sarcophages byzantins monumentaux du vi ${ }^{\mathrm{e}}$ siècle ont été volontairement cassés par des groupes islamistes radicaux pour en détruire les croix. À Shash Hamdan, un tombeau rupestre de grande taille, probablement celui d'une femme appartenant à une famille princière de la région, avait été, lui aussi, vandalisé par des islamistes locaux. Ce magnifique et vaste édifice, creusé dans la falaise qui domine le lac de barrage de l'Euphrate, est l'un des rares monument à témoigner en Syrie de la fin de l'époque hellénistique et des débuts de l'époque romaine. Les bas-reliefs qui ornaient trois des parois de la tombe sur deux niveaux, avec plus de soixante statues de femmes en procession grandeur nature dans des entrecolonnements, un taureau et des cerfs, ont été martelés après que la lourde grille cadenassée de l'entrée a été brisée (fig.6).

Face à la menace de destructions ou de pillages, des tentatives de protections avaient pourtant été mises en œuvre en Syrie. Dans les zones où le service de la DGAM (Direction générale des antiquités et des musées) conserve encore des inspecteurs et des gardiens, des opérations de sauvegarde et de mise à l'abri des collections ont été effectuées, parfois au péril de la vie de leur acteurs. C'est ainsi que les accès aux hypogées de Palmyre avaient été comblés de terre, les portes des tours murées. Des murs de protection avaient été élevés en avant des façades des monuments, et des opérations d'évacuation et de stockage dans des lieux sûrs et protégés avaient été réalisées pour les collections des 34 musées syriens. Cela n’a malheureusement pas empêché que certains objets disparaissent, parfois sans effraction, comme la statuette en bronze dorée de Baal du musée de Hama, non retrouvée à ce jour, et que 

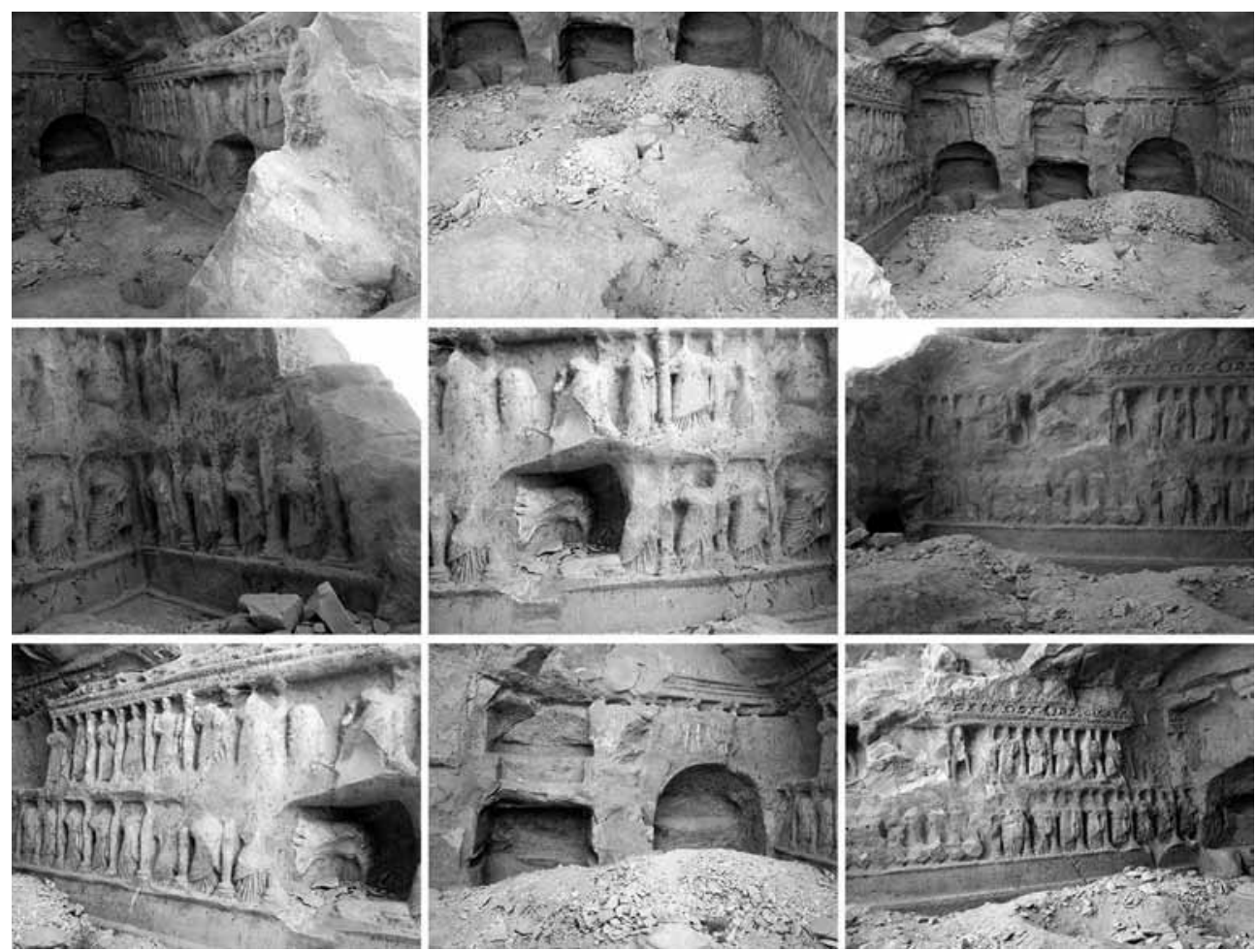

Fig. 6 - Shash Hamdan (Syrie). Destructions dans le tombeau du i ${ }^{\text {er }}$ siècle.

les objets les plus lourds, intransportables, soient détruits comme le lion d'Allat dans le jardin du musée de Palmyre ${ }^{4}$. Outre les efforts des responsables locaux, des initiatives individuelles ou collectives dans les villages ont contribué à mettre des objets en lieux sûrs, à sensibiliser la population à la protection de leur patrimoine local, à faire des photos ou des relevés des bâtiments. Il n'y a jamais eu autant d'images et de vidéos donnant des informations sur l'état du patrimoine au niveau du sol, relayées par les réseaux sociaux via des organismes non gouvernementaux comme l'APSA 2OII (Association for the Protection of Syrian Archaeology). Car si les images satellites diffusées et analysées par unosat (United Nations Operational Satellite) donnent des vues aériennes des dégâts, elles ne permettent pas toujours de savoir ce qui s'est passé à l'intérieur du bâtiment et quels sont les réels impacts des bouleversements sur le terrain. L'American Association for the Advancement of Science (AAAs) en

4 Aucune photo n'a circulé qui confirmerait la destruction de cette grande statue, autrefois placée devant la façade du temple d'Allat à Palmyre. L'information a été transmise à la DGAM par un habitant de la ville. 
partenariat avec des universités et des associations culturelles américaines diffuse et analyse des images sur les six sites syriens classés au patrimoine mondial. L'Asor (American School of Oriental Research), l'APSA et la DGAM fournissent régulièrement des rapports sur l'état des sites. Une liste rouge des biens culturels syriens en péril, susceptibles d'être vendus sur le marché de l'art, a été publiée par l'ıcom (International Council of Museums) et circule largement ${ }^{5}$. Les grandes maisons de ventes aux enchères comme Christie's et Sotheby's se sont engagées à ne vendre aucune antiquité dont l'origine ne soit formellement identifiée en collaboration avec INTERPOL. Car les destructions spectaculairement mises en scène cachent souvent un trafic très fructueux d'antiquités volées.

Dans la zone que contrôle Daech, ces opérations de pillage sont minutieusement orchestrées, soit que l'organisation délivre des «permis de fouille» officiels à des chefs de tribus locales, soit qu'elle se livre directement, avec ses propres "archéologues », au trafic de statues, fresques, monnaies, bijoux etc., soit encore qu'elle prélève un impôt (on parle de $20 \%$ ) sur la vente des objets par les particuliers. Ce trafic alimente grassement ses caisses en plus de ce que lui rapportent le pétrole et le gaz, le trafic de drogues, d'armes et d'esclaves. Les revenus du trafic d'antiquités se monteraient pour l'Iraq et la Syrie à plusieurs centaines de millions de dollars par an 6 . Pour l'instant, peu d'antiquités ont été proposées sur le marché de l'art, soit qu'elles aient été vendues directement à des collectionneurs, soit qu'elles attendent dans des entrepôts au Liban, en Turquie où Dyarbekir est un des lieux du trafic, en Jordanie ou à Dubaï, principaux lieux d'exportation depuis le territoire syrien. Mais peut-être sont-elles déjà dans le port franc de Genève ou à Munich, plaque tournante du trafic sous la coupe d'un “cartel turc», avant de rejoindre les usA, l'un des premiers marchés de collectionneurs d'antiquités ${ }^{7}$.

Le I7 novembre 20I5, le président de la République française, François Hollande, a présenté à l'unEsco un certain nombre de décisions visant à protéger le patrimoine et à lutter contre ce trafic d'objets d'art. Un arsenal de mesures ont été proposées qui passe par le renforcement des contrôles douaniers et des sanctions contre ceux qui font commerce de biens culturels ainsi que la création de zones refuges en France pour y déposer les collections en grand danger, une sorte de droit d'asile du patrimoine culturel. Il est aussi proposé de créer un Fonds international de dotation, public et privé, pour renforcer l'efficacité des efforts financiers

5 Une liste identique doit être diffusée s'agissant des biens culturels libyens.

6 L'estimation précise des revenus du trafic d'antiquités est difficile à faire, c'est pourquoi les chiffres peuvent varier de façon considérable, selon que l'on prend un repère annuel, ou global sur les années depuis lesquelles Daech contrôle de grands secteurs de l'Iraq et de la Syrie et parce que ce trafic est en grande partie souterrain.

7 G. Schwartz, “Antique trafic », Le Monde, 3 avril 2or5, p. I6. 
pour l'instant trop dispersés, ainsi que la constitution de listes noires des «paradis du recel », comme cela existe pour les paradis fiscaux.

Malheureusement, nombre de précautions pour protéger les sites et leurs richesses sur place se sont révélées inutiles face à la volonté des hommes de Daech de détruire coûte que coûte tout ce qui est à leurs yeux «idolâtre » et n'est pas strictement rattaché à l'époque du prophète, ce qui explique la destruction aussi de mosquées ou de lieux saints islamiques. L’opinion a pris la mesure de cette volonté mise à exécution en 2014 à la vue d'images révoltantes d'hommes qui saccageaient le musée de Mossoul, renversant des statues monumentales, les détruisant à la masse quand ils ne les faisaient pas exploser à la dynamite. Plus tard, d'autres images ont montré les mêmes scènes à Hatra, à Ninive et des explosions ont détruit des quartiers entiers de l'antique Nimroud. Les images venues d'Iraq ont fait prendre conscience de la détermination de ces adeptes d'un mouvement radical qui veut faire table rase du passé. Au musée de Mossoul, les images de la destruction sauvage des statues assyriennes ont fait le tour du monde, tout comme celles de la destruction au marteau-piqueur du grand taureau ailé à tête humaine de Ninive (vıI ${ }^{\mathrm{e}}$ s. av. J.-C.) qui flanquait la porte de Nergal (fig. 7). Trop lourdes ou trop fragiles, ces sculptures avaient dû être laissées sur place, à la différence des petits objets transportés à Bagdad avant 2003. À Hatra, les vestiges des $\mathrm{I}^{\mathrm{er}} \mathrm{s}$. av. et $\mathrm{I}^{\mathrm{er}} \mathrm{s}$. ap. J.-C. ont été attaqués à la masse, à la pioche et, pour les plus hauts, à la kalachnikov, les hauts-reliefs étant abattus parce qu'ils représenteraient «des idoles». Se déclarant fidèles à Mahomet, les djihadistes se vantent de détruire tout ce qu'ils assimilent à des manifestations de polythéisme. Le is avril, une nouvelle vidéo a révélé la destruction à l'explosif du palais nord-ouest de Nimroud datant du règne d'Assourbanipal II (883-869 av. J.-C.). Les bas-reliefs qui ornaient les murailles, arrachés de leur support, furent évacués au bulldozer et des statues colossales de taureaux ailés détruites, avant qu'une explosion vienne ensuite détruire le palais.

L'œuvre de destruction de Daech n'a pas épargné la Syrie. À leur arrivée sur l'Euphrate, ses hommes ont détruit volontairement plusieurs mausolées à Raqqa dont deux étaient ceux de martyrs de la bataille de Siffin en 657 qui opposa Ali à Muwayah pour le califat, Uwais al-Qarani et Ammar bin Yasser. Mais ce qui a le plus frappé et bouleversé l'opinion internationale, c'est leur acharnement contre l'un des sites les plus emblématiques de la Syrie, celui de Palmyre. L'arrivée des hommes de Daech à Palmyre le 2ımai 20ı5, depuis Raqqa ou Deir ez-Zor sur l'Euphrate, après une traversée du désert de près de $300 \mathrm{~km}$, sans qu'ils soient le moins du monde arrêtés ou combattus, a suscité immédiatement les plus vives inquiétudes. Il n'a malheureusement pas fallu attendre longtemps pour se rendre compte que les craintes n'étaient pas vaines. En effet, moins d'une semaine plus tard, le théâtre de la cité antique servait de “décor» à une exécution de masse de 
soldats du régime prisonniers. Le ı8 août, une vidéo diffusait les images du corps décapité et mutilé de Khaled al-As'ad, l'ancien directeur du site accusé d'“avoir fréquenté des infidèles» lors de colloques à l'étranger et d'être le "gardien des idoles ». Le 23 août, le temple de Baalshamin explosait (fig.8), une semaine plus tard c'était le tour du grand temple de Bêl (fig.9), suivi par la destruction totale de plusieurs tours funéraires parmi les mieux conservées et les plus belles, celles de Jamblique et d'Elhabel, où subsistaient en outre des traces de peinture ${ }^{8}$, et de celle du grand arc quelques jours plus tard.

Ces actes ont été qualifiés à juste titre de « nettoyage culturel », de « furie destructrice» par Irina Bokova, directrice de l'unesco. Ils appellent, selon elle, à «une réaction de la Cour pénale internationale», ce que ne dément pas Nabil al-Arabi, secrétaire général de la Ligue arabe qui parlait «d'agression barbare contre le patrimoine du peuple irakien» et de “crimes les plus abominables commis en notre temps contre le patrimoine de l'humanité».

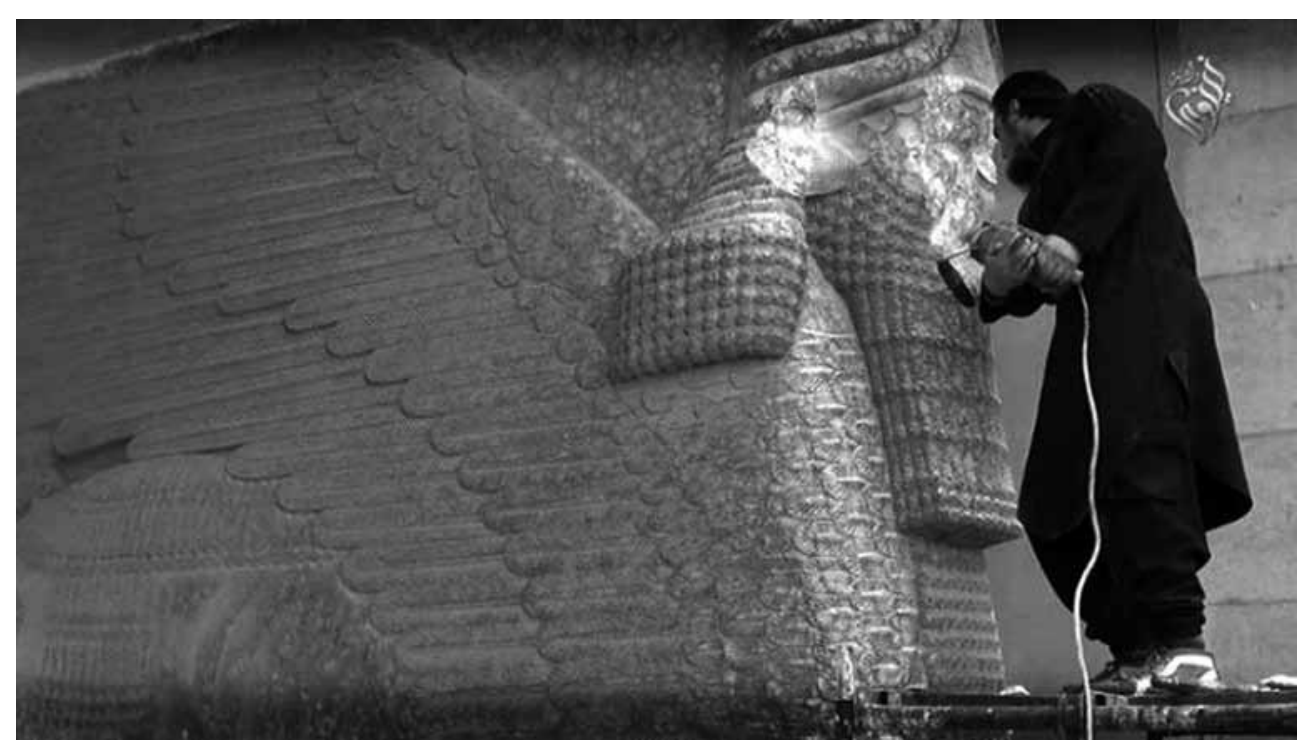

Fig. 7 - Mossoul. Mutilation du grand taureau ailé de Ninive (viI ${ }^{\mathrm{e}}$ siècle av. J.-C.).

8 Parmi les tours détruites outre celles d'Atenatan, la plus anciennement datée de 9 av. J.-C. et celle d'Elhabel de ıo3 ap. J.-C., situées dans la vallée des Tombeaux dans la nécropole ouest, plusieurs tours de la butte de Belqis ont été pulvérisées. Il s'agit de celles de Jamblique de 83 ap. J.-C. et des tours anonymes $\mathrm{n}^{\text {os }}$ 7o et $7 \mathrm{I}$; ainsi que celle de Kithôt de 40 ap. J.-C. située sur la pente sud du Jebel al-Hussayniyeh à l'opposé de la butte d'Umm Belqis. 


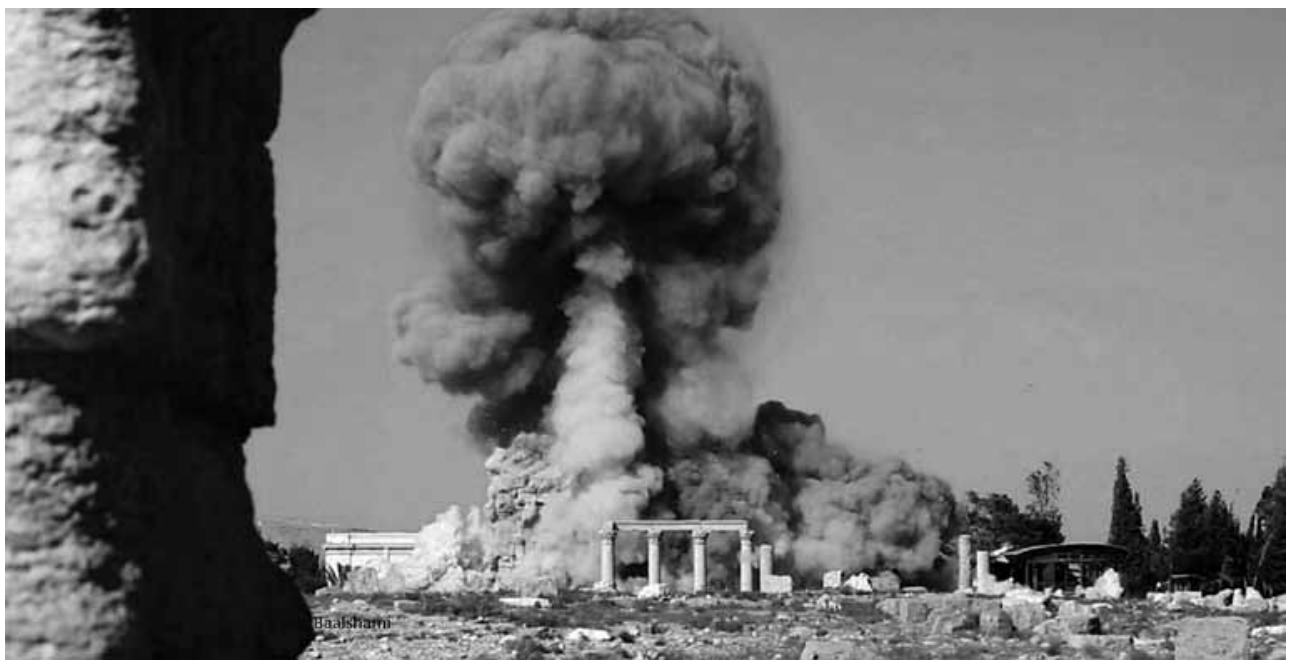

Fig. 8 - Palmyre. Explosion du temple de Baalshamin le 4 août 2015.

En réduisant les deux plus beaux temples de Palmyre et les tours funéraires à des tas de gravats, Daech a non seulement totalement défiguré le site, mais il a effacé du paysage les traces d'une histoire millénaire et les témoins de la fusion des cultures gréco-romaine et syro-mésopotamienne qui étaient si présentes sur l'ensemble du site. Qu'est-ce que Palmyre aujourd'hui sans son gigantesque temple de Bêl qui fut successivement un temple des dieux palmyréniens (Bêl, Malakbêl, Yahribôl, Aglibôl, Astartè, Manawat, Herta, etc.), des dieux grecs ou assimilés (Héraclès, Mars-Arsu, Vénus-Atargatis), puis une église ${ }^{9}$ et une mosquée, celle des villageois installés dans l'enceinte jusque dans les années I930 ${ }^{10}$ ? Qu'est-ce que Palmyre sans ses tours funéraires emblématiques du site, mémoires de ces riches caravaniers réunis là avec toute leur lignée pour l'éternité et qui, sur les bas-reliefs des plaques de loculi ou les sarcophages, de leur regard déjà tourné vers

9 Il subsistait sur le mur intérieur est du temple une fresque représentant la Vierge avec l'enfant entourée de deux saints portant une couronne et des palmes, ainsi qu'un graffiti en grec émanant d'un certain Lazare honorant la "sainte Mère de Dieu », daté du $\mathrm{VI}^{\mathrm{e}}$-vII ${ }^{\mathrm{e}}$ siècle. Cf. E. Jastrzebowska, “La christianisation de Palmyre: l'exemple du temple de Bel », Studia Palmyrenskie, XII, 20I3, p. I77-I9I, et J.-B. Yon, IGLS XVII, вAH I95, Beyrouth, 2012, $\mathrm{n}^{\circ} 47$.

10 Des photographies aériennes montrent l'installation du village dans le temenos du temple qui servait de mosquée aux habitants. Le déménagement du village se fit en I929 à l'initiative des archéologues français qui entreprirent de dégager le temple, de l'étudier et de le restaurer. La publication finale de ces travaux est due à H. Seyrig, R. Amy et É. Will, Le temple de Bêlà Palmyre, ван 83, Paris, I968 et 1975. 
l'au-delà, nous faisaient face parés de leurs plus beaux atours et bijoux? Qu'est-ce que Palmyre sans son petit temple de Baalshamin, admirable de proportions et où s'affichait fièrement en grec et en araméen sur l'une des consoles de la façade le nom de celui qui l'avait rénové et embelli, Malè Agrippas? Ce bienfaiteur et évergète rappelait également qu'il avait fourni l'huile (pour le gymnase) à l'empereur Hadrien et à toute sa délégation lors de la venue de celui-ci dans l'oasis en i3o ap. J.-C. ${ }^{1}$. C'est sans doute pour remercier de cela que l'empereur autorisa la cité à s'appeler désormais Hadriana Palmyra.

Savaient-ils les sauvages qui les ont détruits combien tous ces monuments étaient indispensables à l'histoire du site et des hommes qui avaient vécu là il y a deux mille ans? Peut-être. Et peut-être savent-ils aussi qu'en pillant et fouillant sans relâche les sols pour y trouver des objets de commerce, ils détruisent à tout jamais l'espoir de faire progresser notre connaissance de cette civilisation. 8o \% du site de Palmyre étaient encore inexplorés en 20II, et les fouilles commencées au sud du wadi dans la partie la plus ancienne promettaient de belles découvertes sur la Palmyre hellénistique. En deux sondages effectués entre I997 et 20IO, il y fut découvert des rues, des maisons à péristyle ornées de peintures et de stucs, des bâtiments publics et une foule d'objets de la vie quotidienne ou reliés au commerce lointain comme des amphores vinaires, des céramiques locales et d'importation, des bronzes. La poursuite de ces fouilles aurait pu nous révéler l'aspect de cet espace urbain, occupé depuis le $\mathrm{II}^{\mathrm{e}}$ siècle av. J.-C. et partiellement abandonné au III $^{\mathrm{e}}$ siècle ap., et peut-être confirmer ou infirmer l'hypothèse qu'il fut en partie détruit lors de la prise de la ville par Aurélien en $27^{3}{ }^{12}$. L'ignorance du contexte des trouvailles clandestines, leur dispersion et l'impossibilité de les relier à des séries interdiront sans doute à tout jamais d'en étudier le sens. C'est bien à la mort d'un site exceptionnel et du patrimoine d'un pays et de l'humanité entière que nous assistons impuissants. Car que peut-on faire tant que la guerre durera et que les destructeurs de tous bords persisteront à occuper et à se battre dans les lieux historiques et sur les sites archéologiques? L'escalade de la violence sur place et la multiplication des acteurs du conflit sur le sol même de la Syrie depuis 20II ne permettent pas à l'heure actuelle de fixer un terme à cette situation et les bombardements quotidiens sur l'ensemble du territoire n'épargnent ni les hommes ni les pierres. L'élimination des éléments les plus violents qui se livrent à la destruction volontaire, en l'occurrence Daech, devrait peut-être

1 Cf. J.-B. Yon, Inscriptions Grecques et Latines de Syrie, XVII/I, вAн I95, Beyrouth, 20I2, nI 45 , et P. Collart, J. Vicari, Chr. Dunand, R. Fellmann, Le sanctuaire de Baalshamin à Palmyre, Rome, 1969-2000.

12 A. Schmidt-Colinet et W. el-As'ad, Palmyras Reichtum durch Weltweiten Handel, Archäologiche Untersuchungen im Bereich der hellenistischen Stadt, Wien, 2013. 
permettre de mettre un frein aux déprédations et sauver ce qui reste à Palmyre ou dans les autres sites historiques tenus par l'organisation. Mais la stratégie utilisée jusqu'à présent, d'intervenir uniquement par voie aérienne, favorise les risques de dommages collatéraux et, de l'avis des spécialistes, est insuffisante pour éliminer ou chasser totalement Daech de Syrie.

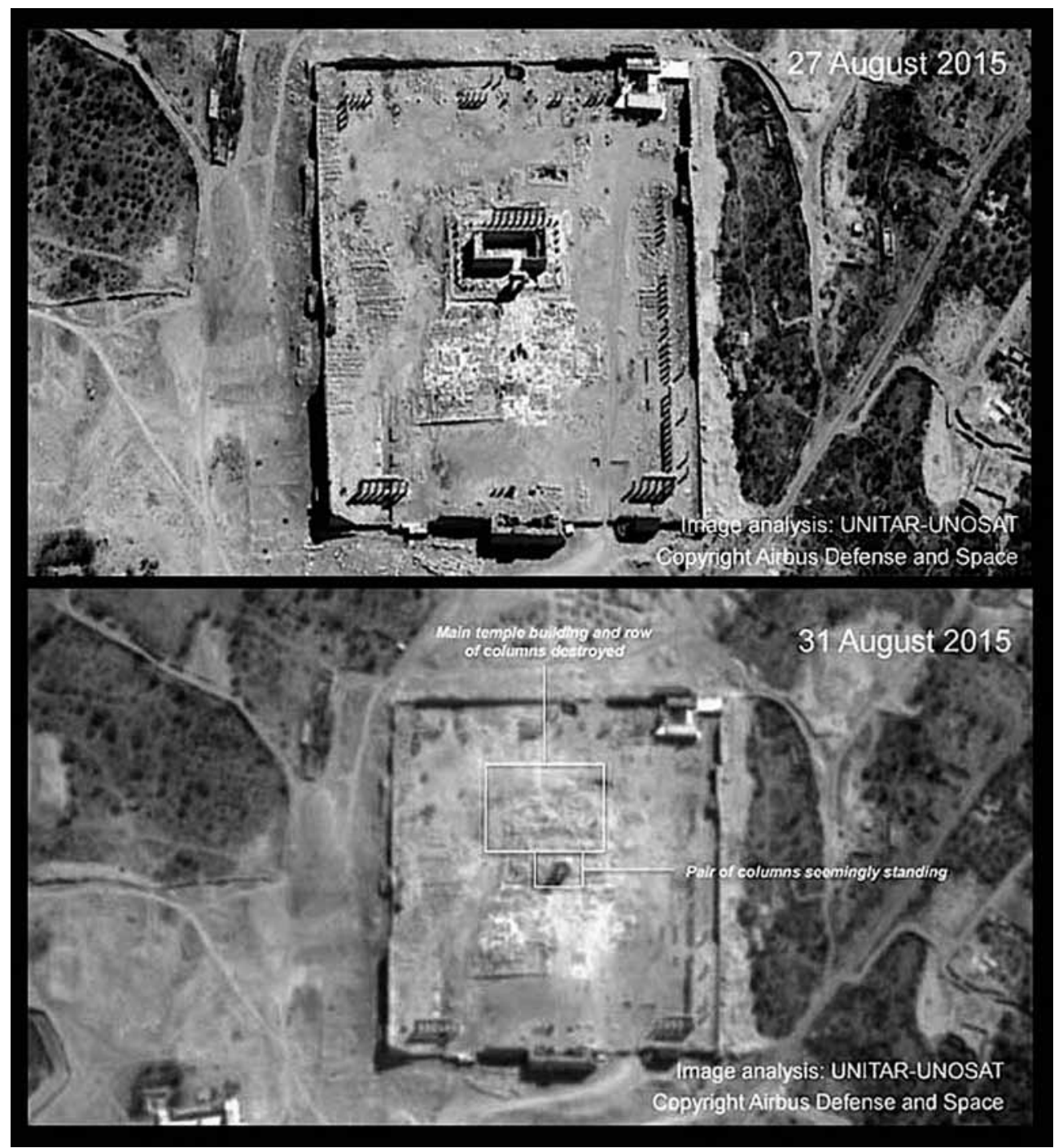

Fg. 9 - Palmyre. Le temple de Bêl, vues aériennes des 27 et 3ı août 20 I5. 
Malgré cette incertitude sur la durée du conflit, on doit néanmoins se poser la question de savoir comment envisager l'avenir pour le jour où la paix reviendra, s'il reste encore quelque chose à préserver. Un état des lieux s'imposera avec un inventaire précis des dégâts et une évaluation des possibilités de restauration et de reconstruction. Sous le coup de l'émotion, on évoque ici et là la reconstruction des temples de Baalshamin et de Bêl, mais est-ce bien raisonnable? Là où les pierres d'origine existent, comme cela semble être le cas pour le grand arc, peut-être peuton envisager une anastylose, mais à quoi rimerait de reconstruire à neuf le temple de Bêl? S'agirait-il en outre de le reconstruire comme il était avant septembre 2or5, ou de le refaire dans son état d'origine quand il fut totalement achevé en i 75 ap. J.-C. ${ }^{13}$ ? Comment être sûr que le site ne va pas se transformer en Disneyland, ce qui reviendrait à le faire mourir une fois de plus? Ce qui faisait le charme des lieux, c'était leur accès libre de jour comme de nuit; le souci de protéger Palmyre ne doit pas conduire à imiter ce qui s'est fait sur le site antique de Jérash, entouré de murs et de grilles et accessible seulement après la traversée de souqs où les « marchands du temple» vendent le meilleur parfois, mais surtout le pire.

L'ensemble de la communauté scientifique doit néanmoins continuer à se mobiliser pour appeler à sauver Palmyre de ceux qui la détruisent aujourd'hui, mais aussi de ceux qui seraient tentés de la défigurer plus tard. Tout ceci est valable pour l'ensemble des sites qui ont souffert de la guerre, c'est pourquoi il faut rester prudent et modérer les tentations que l'on peut avoir, pour effacer au plus vite les désastres, de restaurer hâtivement avec des matériaux inadéquats et dans l'irrespect du bâti ancien, comme on a pu le voir parfois sur bien des sites du Hauran où le parpaing et le béton suppléaient le basalte d'origine. Sans doute, le plus urgent est-il de former dès aujourd'hui en France et ailleurs auprès des grandes institutions culturelles compétentes des spécialistes, des restaurateurs, des gens instruits des diverses techniques et de l'histoire dont le souci devra être de respecter le passé et non pas de l'exploiter en vue d'un profit financier immédiat. C'est pourquoi il est important d'accueillir des archéologues iraquiens ou syriens dans les établissements culturels ou de recherche français. Il est urgent de les former, ainsi que les doctorants, afin de constituer un corps d'experts pour demain dans leur pays d'origine, à charge pour eux de répercuter ce savoir sur place auprès des archéologues, des personnels des musées et des gardiens

13 Il est communément dit que le temple fut consacré en 32 ap. J.-C., mais il ne s'agissait là que de l'achèvement d'une partie du temple (le thalamos nord), commencée une quinzaine d'années plus tôt, ce dont témoignent plusieurs inscriptions entre ig et 28 ap. J.-C. (IGLS XVII, 24, I6, I7). Deux inscriptions, datées respectivement de io8 (IGLS XVII, 23) et de i75 ap. J.-C. (IGLS XVII, 2I), commémorent les dons de portes de la cella et de l'entrée du péribole et datent sûrement l'achèvement final. 
de sites. On ne devrait plus voir en Syrie de fouilles sans publication rapide des découvertes et du matériel, plus de musées sans livres d'inventaires sérieux, plus de responsables du patrimoine sans connaissance des richesses patrimoniales du terrain, non seulement dans les grands sites, mais aussi dans les moindres villages qui, en Syrie, recèlent tous quelque chose du passé. Il ne suffira pas de faire des lois, mais de les faire respecter par tous sans exclusive, et d'éradiquer les pratiques de corruption et d'intimidation de toute sorte. Et enfin, à la base de tout, concevoir un enseignement digne de ce nom de l'histoire de ces pays, débarrassé des préoccupations nationalistes ou religieuses qui conduisent à valoriser, ou au contraire à dénigrer telles ou telles périodes, à laisser croire que tous les habitants sont les descendants des conquérants musulmans du viI ${ }^{\mathrm{e}}$ siècle, alors qu'en réalité ils sont, dans leur grande majorité, les descendants de ceux qui ont laissé leur trace culturelle dans ce pays qui, de tout temps, a vu passer et accueilli les civilisations antérieures. Il ne devrait plus y avoir personne pour dire devant tel ou tel monument “ce n'est pas mon histoire pour en justifier le désintérêt ou la destruction. Tout vestige du passé dans un pays quel qu'il soit est un témoin d'un moment de l'humanité, le nier c'est nier ce que l'on est soi-même.

\author{
Annie Sartre-Fauriat \\ Professeur des Universités émérite \\ CREHS Université d'Artois (EA 4027) \\ HISoMA Lyon (UMR 5189) \\ 30 , rue des Perriers \\ 37170 Chambray-lès-Tours \\ 0033 (0)680342482 \\ asartre37@gmail.com
}

